\title{
THE DIFFERENTIAL POLARIZATION OF \\ STARLIGHT AS A FUNCTION OF DISTANCE
}

\author{
STEPHEN LLOYD and MARTIN O. HAR WIT \\ Center for Radiophysics and Space Research, Cornell University, Ithaca, N.Y. 14850, U.S.A.
}

\begin{abstract}
We have plotted the differential polarization as a function of distance and galactic longitude for 1732 stars between galactic latitudes $-5^{\circ}$ and $+5^{\circ}$. The data were obtained from several published sources. A fairly clearcut distinction is seen between the effects of spiral arms and inter-arm regions indicating that starlight is systematically polarized only when passing through spiral arms.
\end{abstract}

One reason why the polarization of starlight is interesting is that it gives us information about two different phenomena, both related to the galactic structure. The first is the distribution of dust; the second is the mechanism that aligns the dust grains.

We have determined the variation of the polarization with distance. The goal was to find what we shall call the incremental polarization at a given distance and longitude in the galactic plane. This incremental polarization is the average polarization of the stars in a given area of the galactic plane with the foreground polarization subtracted. It represents the local polarization intrinsic to the area.

In order to do this, we have gathered optical polarization data from a number of sources, principally Hall's (1958) catalog of northern stars and Mathewson and Ford's (1970) catalog of southern stars (see also Appenzeller, 1966 and Behr, 1959). In all, we used 1732 stars between galactic latitudes $-5^{\circ}$ and $5^{\circ}$. These stars were divided into 180 sectors in the galactic plane. The plane was divided first into 36 longitude intervals, each $10^{\circ}$ wide; then each longitude interval was divided into 5 distance intervals: 0-400 pc, $400-800 \mathrm{pc}, 800-1500 \mathrm{pc}, 1500-2500 \mathrm{pc}$, and $>2500 \mathrm{pc}$.

In order to add or subtract polarizations, we made use of the Stokes parameters $Q$ and $U$ for each star. Then, for each sector of space, we averaged $Q$ and $U$ over all the stars in that sector. Finally, from the average values of $Q$ and $U$ in a sector $B$, we subtracted the average values of $Q$ and $U$ in the adjacent nearer sector $A$ at the same longitude. From these difference values, $Q_{B}-Q_{A}$ and $U_{B}-U_{A}$, we computed the incremental polarization and polarization angle corresponding to sector B. Actually, this incremental polarization refers to a distance interval that overlaps $A$ and $B$, rather than just to $B$. Unfortunately, this does not always work well. There usually is a large dispersion in the polarization and sometimes in the angle, so that often the dispersion in $Q$ and $U$ is as large as the differences $Q_{B}-Q_{A}$ and $U_{B}-U_{A}$.

In Figure 1 we see some of the results. Each column is one longitude interval. The nearest distance interval is at the top; the farthest at the bottom. As is customary, the length of each line is proportional to the incremental polarization; a vertical line represents a polarization angle of $0^{\circ}$; a horizontal line - an angle of $90^{\circ}$. We can see how both the magnitude and the angle of the local incremental polarization vary with distance. 
For example, at $l=80^{\circ}$ and $l=90^{\circ}$ we see that the incremental polarization is moderately large out to great distances, while at $l=100-120^{\circ}$ we see the polarization dropping off in the most distant intervals. At $l=140^{\circ}$, in Figure 2, we see the polarization drop off and then increase again; the large polarization in the most distant

$$
\text { DIFFERENTIAL POLARIZATION, }-5<6^{\mathrm{II}}<5
$$

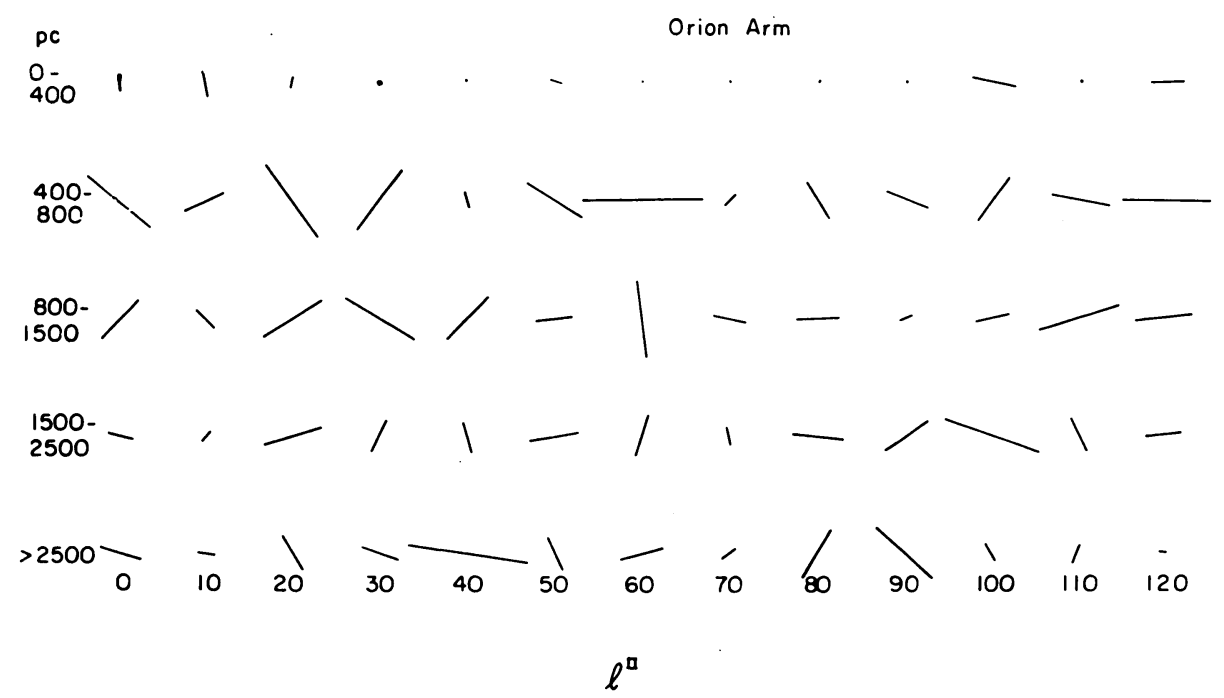

Fig. 1. The differential polarization of starlight in the galactic plane as a function of distance and longitude. The length of the vector at $l=0^{\circ}, 800-1500 \mathrm{pc}$, represents a polarization of $1 \%$. A vertical vector represents a polarization angle of $0^{\circ}$.

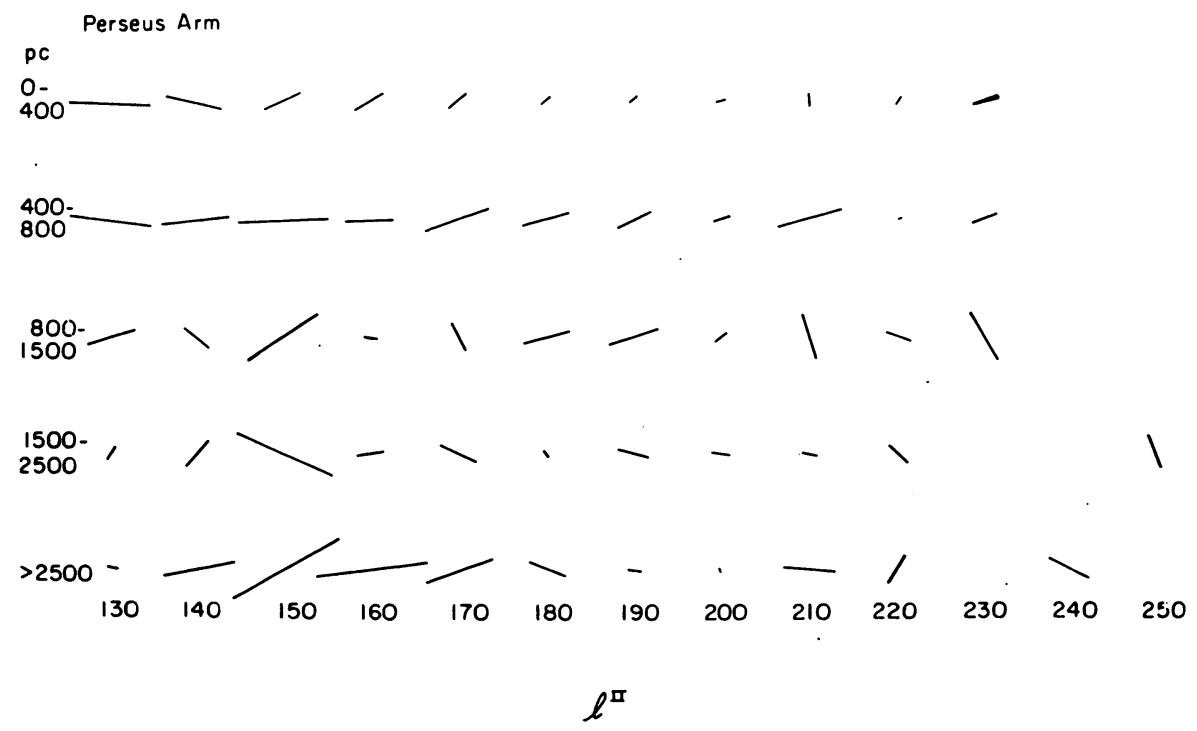

Fig. 2. See caption to Figure 1. 
intervals between $l=140^{\circ}$ and $l=170^{\circ}$ may be due to the presence of the Perseus Arm. Around $l=130^{\circ}$, the polarization vectors are most closely aligned to $90^{\circ}$. The polarization here is somewhat larger than in the opposite direction at $l=310^{\circ}$. Here we see rather weak polarization, perhaps because the Sun is nearer the inside edge of the local arm. Figure 3 shows a region, in which statistics are somewhat poor.

In Figure 4 we see a new way of presenting the polarization data in the galactic plane. We have taken the polarization vectors from the preceeding figures and put them onto a chart of the local spiral arms. The stars and crosses represent clusters and associations of supergiants; this chart was taken from a recent review paper by Bok (1970).

Each polarization vector was placed halfway between the middle of the distance intervals used in its computation. Together the vectors form rings at distances from the Sun of $400,900,1600$, and $2500 \mathrm{pc}$. The rings containing the polarization vectors were placed at distances from the Sun roughly representative of their distance interval $600 \mathrm{pc}, 1200 \mathrm{pc}, 2000 \mathrm{pc}$, and $2500 \mathrm{pc}$. The innermost distance group was omitted. A vector parallel to the tangent of the circle means a polarization angle of $90^{\circ}$.

The first thing we notice is the familiar strong alignment around $l=130^{\circ}$, and, to a lesser extent, $l=310^{\circ}$. Also, the incremental polarization seems substantially larger at the $600 \mathrm{pc}$ ring than further out. Along the ends of the local arm, the incremental polarization is generally weak, although the angle is not highly scattered.

In the Orion Sagittarius interarm region the angle is highly scattered, as one would expect. The incremental polarization seems moderately high, although this may be a spurious effect of the large angle scatter.

Around $l=150^{\circ}$ we see a large incremental polarization that might be evidence of the Perseus Arm. The polarization in that arm around $l=120^{\circ}$, however seems anomalously low. The Sagittarius Arm may be 'hidden' by the large angle scatter in the

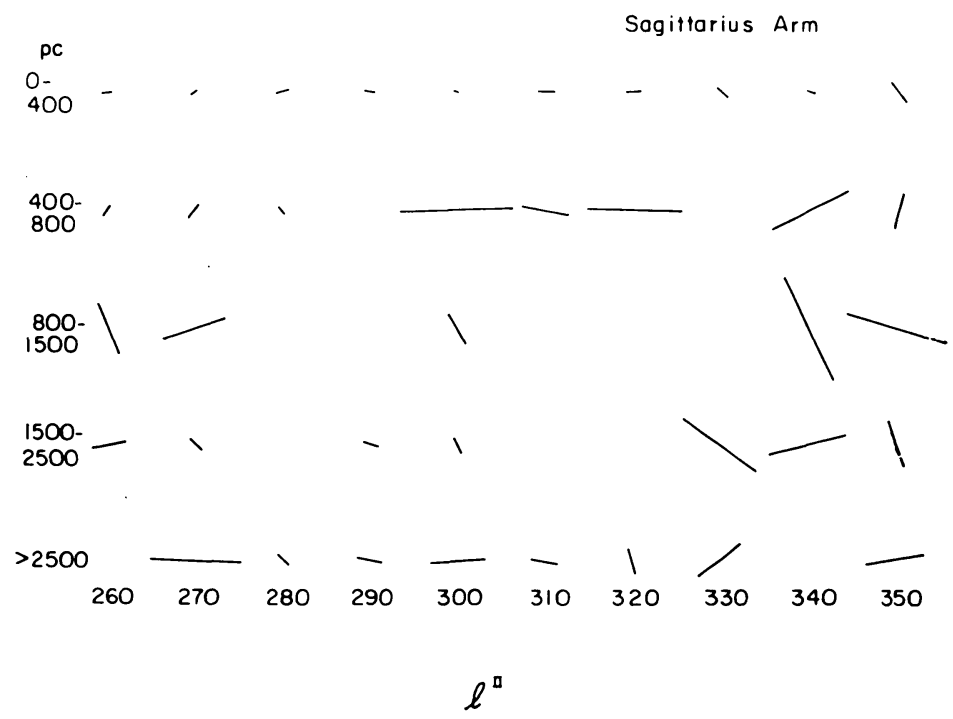

Fig. 3. See caption to Figure 1. 


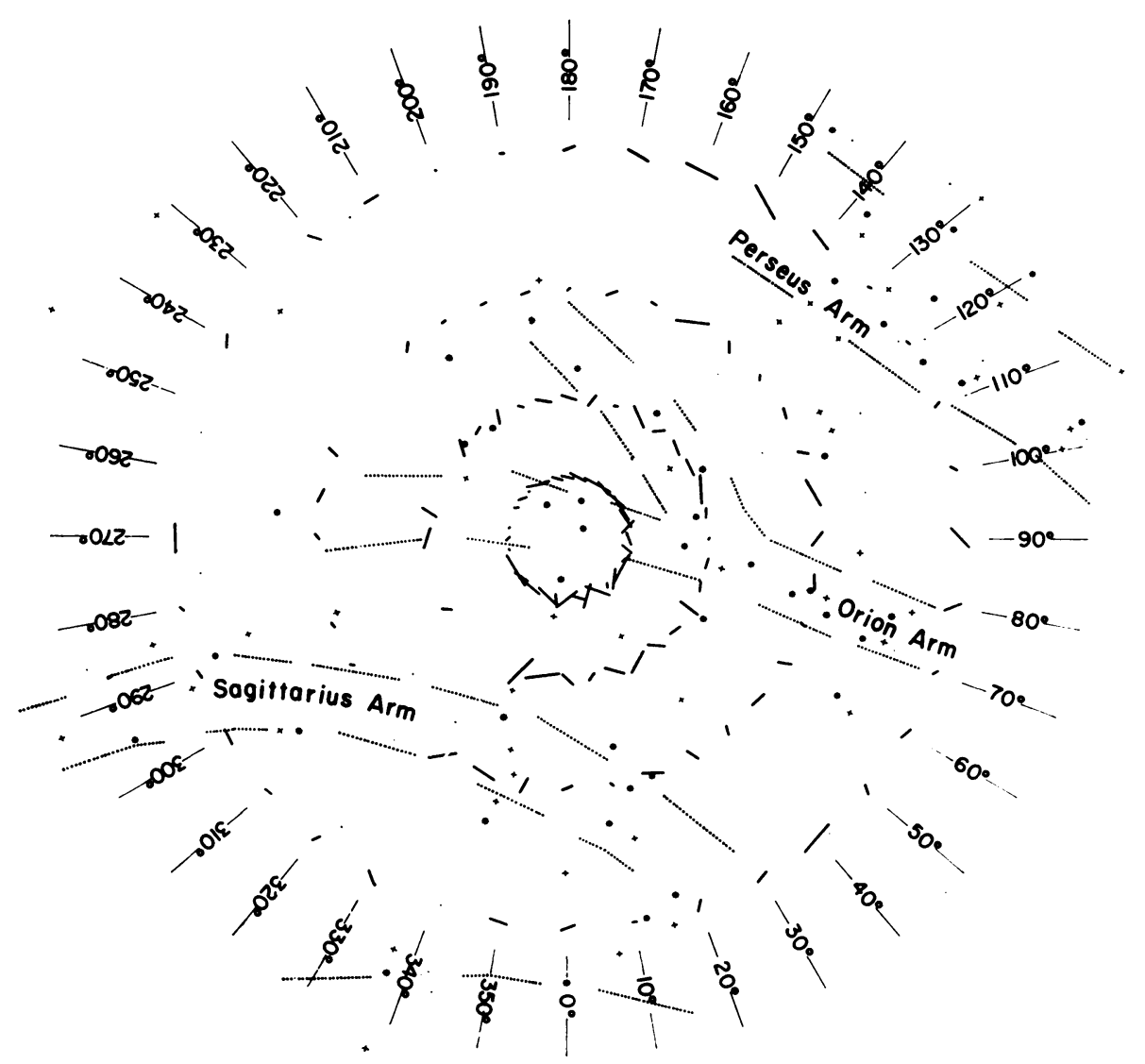

Fig. 4. The differential polarization vector displayed on a chart of the local spiral arm structure. The outer ring has a radius of $2500 \mathrm{pc}$.

interarm region, although between $l=340^{\circ}$ and $350^{\circ}$, at $\approx 2 \mathrm{kpc}$ we see some alignment.

Whatever the case, these are just the first results, and we expect that as this technique of finding the incremental polarization is developed, it will begin to yield more useful new evidence about the distribution and alignment of dust in our part of the Galaxy.

\section{Acknowledgements}

This work was supported by the Research Corporation. One of us S. L. was supported by the Ford Foundation through the 6-yr PhD program at Cornell University.

\section{Appendix}

The Stokes parameters are characteristics of light beams, not polarizers. The following argument is intended to justify the use of the Stokes parameters (in this paper to 
describe polarizing media. (In this discussion, the fourth Stokes parameter, $V$, is ignored).

If a beam of unpolarized light with Stokes vector $\bar{S}_{i}=\left(I_{i}, 0,0\right)$ is incident on a polarizer, the emerging beam is $\bar{S}_{0}=\left(I_{i} /\left(1+P_{p}\right)\right)\left(1, q_{p}, u_{p}\right)$, where $P_{p}$ is the polarization degree of the emerging beam, $q_{p}=P_{p} \cos 2 \theta_{p}, u_{p}=P_{p} \sin 2 \theta_{p}$, and $\theta_{p}$ is the angle of polarization of the emerging beam.

Consider a weakly polarized beam

$$
\bar{S}_{i}=I_{i}\left(1-P_{i}, 0,0\right)+I_{i}\left(P_{i}, q_{i}, u_{i}\right) \quad P_{i} \ll 1
$$

incident upon a weak polarizer $\left(P_{p} \ll 1\right)$. To the first order in $P_{i}$ and $P_{p}$, the second, polarized component of $\bar{S}_{i}$ is transmitted unchanged by the polarizer. The emerging beam is then

$$
\bar{S}_{0}=\left(I_{i}\left(1-P_{i}\right) /\left(1+P_{p}\right)\right)\left(1, q_{p}, u_{p}\right)+I_{i}\left(P_{i}, q_{i}, u_{i}\right) .
$$

Using $\left(1-P_{i}\right) /\left(1+P_{p}\right) \approx\left(1-P_{i}\right)\left(1-P_{p}\right) \approx 1-P_{i}-P_{p}$ and ignoring second order terms, we find

$$
\bar{S}_{0}=I_{i}\left(1-P_{p}-P_{i}, q_{p}, u_{p}\right)+I_{i}\left(P_{i}, q_{i}, u_{i}\right) \sim I_{i}\left(1, q_{p}+q_{i}, u_{p}+u_{i}\right) .
$$

\section{References}

Appenzeller, I.: 1966, Z. Atrophys. 64, 296.

Behr, A.: 1959, Veröff. U. Sternw. Göttingen, No. 126.

Bok, Bart J.: (1970) in De Jager (ed.), Highlights of Astronomy, p. 63.

Hall, J. L.: 1958, Publ. U.S. Naval Obs. 2nd Ser. 17, No. VI.

Mathewson, D. S. and Ford, V. L.: 1970, Monthly Notices Roy. Astron. Soc. 74, 139. 